\title{
Strain Development for Water Assimilable Organic Carbon Detection
}

\author{
Xingding Zhou ${ }^{1}$, Haibao Zhang ${ }^{2}$ \\ ${ }^{1}$ School of Life Sciences \& Chemical Technology, Ngee Ann Polytechnic \\ 535 Clementi Road, Singapore \\ ZHOU_xingding@np.edu.sg \\ ${ }^{2}$ Water Quality Office, Public Utilities Board \\ 82 Toh Guan Road East Singapore \\ ZHANG_Hai_Bao@pub.gov.sg
}

\section{Extended Abstract}

Assimilable organic carbon (AOC) refers to a fraction of biodegradable dissolved organic carbon that is readily assimilated by microorganisms for cell growth. It consists of a broad range of low molecular weight organic carbon molecules such as sugars, organic acids, and amino acids. AOC is a critical parameter for drinking water treatment and distribution processes. Several studies have linked AOC and biofilm formation in drinking water distribution systems [1, 2]. Thus AOC test is a standardized measure of the bacterial regrowth potential of treated water.

Conventional AOC analysis is done with a bioassay that was originally developed by Dutch researcher Van der Kooij and co-workers and later adapted by others as stated in the Standard Methods[3-4]. This method basically contains two stages, cell culturing and cell enumeration. In the cell culturing stage, the water sample is inoculated with two water representative strains (Pseudomonas fluorescens P-17 and Spirillum sp. NOX), and incubated at $15{ }^{\circ} \mathrm{C}$ for 9 days to reach the stationary phase. On days 7,8 and 9, culture cell enumeration is determined by traditional plating counting on nutrient agar, which required another 3 - 5 days. Finally the cell numbers of the two strains are converted to AOC amount by previously derived empirical yield values. This method is tedious, labour-intensive and notably time-consuming (>14 days). Thus, a fast and easily applicable AOC testing method is essential for an efficient water treatment system and water bio-stability monitoring.

The long incubation time in the first stage is caused by the slow growth rate of Pseudomonas fluorescens $\mathrm{P}-17$ and Spirillum sp. NOX strains. Rapid growth strains can be successfully obtained by evolutionary engineering. Classical mutagens, UV, heat shock are robotic, newly developed methods like genome shuffling and transcription factor optimization further strengthen the tools for strain improvement by evolutionary engineering. In this work, we have obtained the fast growing strains without compromising of the carbon source utilization spectrum, thus we could dramatically reduce the time for the first stage.

The long process time is an inherent disadvantage for plate counting. A more robotic method should be applied. Flow cytometry has emerged as the leading tool for single-cell analysis. One of the advantages of flow cytometry is that it allows for rapid and accurate enumeration of different types of cells in a cell mixture. In this work, we have demonstrated that using flow cytometry can reduce the total timing for AOC detection.

\section{References}

[1] I. C. Escobar, A. A. Randall, J. S. Taylor, "Bacterial growth in distribution systems: effect of assimilable organic carbon and biodegradable dissolved organic carbon," Environ Sci Technol, vol. 35, no. 17, pp. 3442-3447, 2001.

[2] D. Van Der Kooij, "Assimilable organic carbon as indicator of bacterial regrowth," J. Am. Water Works Assoc., vol. 84, no. 2, pp. 57-65, 1992.

[3] D. van der Kooij, A. Visser, W. A. M. Hijnen, "Detremination of easily assimilable organic carbon in drinking water," J. Am. Water Works Assoc., vol. 74, pp. 540-545, 1982.

[4] L. A. Kaplan, T. L. Bott, D. J. Reasoner, "Evaluation and simplification of the assimilable organic carbon nutrient bioassay for bacterial growth in drinking water," Appl Environ Microbiol, vol. 59, no. 5, pp. 1532-1539, 1993. 